confirmed sarcoidosis attending a special clinic at the Mount Sinai Hospital, New York. Fourteen of the 18 patients were women. The age of all the patients ranged from 13 to 54 years, and the duration of disease at the onset of neurological manifestations had varied from a few days to seven years. Fourteen of the 18 patients had intrathoracic lesions, 11 ocular lesions, 9 superficial lymphadenopathy, 7 salivary-gland enlargement, 5 hepatomegaly, 4 skin lesions, 2 splenomegaly, 2 lacrimal-gland enlargement, and 2 erythema nodosum. The Kveim test was positive in 15 out of 16 patients tested and the tuberculin test (250 T.U.) was negative in 14 out of 17 patients so tested. Sarcoid tissue was found at biopsy in scalene nodes of 6 patients, peripheral lymph nodes of 5 , the skin of 4, the liver of 2 , and muscle, nasal mucosa, bronchus, lung, enucleated eye, or brain of 1 each. The appearance of neurological symptoms or signs during the course of known sarcoidosis was the principal reason for more detailed investigation of the nervous system. Without the knowledge of sarcoidosis in other systems the pathogenesis of the neurological change would have remained obscure. Neurological disease was of three anatomical types: 6 patients had lesions of the central nervous system, 4 had lesions of peripheral nerves, and 8 had lesions of cranial nerves. Facial palsy was present in 12 of the 18 patients and was accompanied by enlargement of the parotid gland in only two.

The diagnostic procedures usual in neurology were unhelpful in the diagnosis of neurosarcoidosis. The cerebrospinal fluid was abnormal in only 5 of 11 studied, and pleocytosis was evident in only two of these. The variable course of the neurological manifestations made it difficult to assess the value of corticosteroid therapy. The course in patients who did not receive steroids was almost as favourable as in those who did.

The patients described in the New York report were included only if they met the rigid diagnostic criteria defined at the International Conference on Sarcoidosis ${ }^{3}$ held in 1960 in Washington, D.C. By contrast, W. B. Matthews's series ${ }^{2}$ of nine patients with sarcoidosis of the nervous system were not selected from a special sarcoidosis clinic but were the patients of one neurologist drawing from a population of about 500,000 over a period of 10 years. Histological proof was obtained in only two-thirds of them and the Kveim test was not done. The British series, like the American, comprised more women than men ( 8 of the 9$)$. Facial palsy and intrathoracic lesions were common, as also were pain and hyperalgesia of a bizarre distribution on the trunk. Indeed, this last complaint led to the provisional diagnosis of pleurisy, appendicitis, and spinal arthritis in three instances.

Sarcoidosis affects more than one system, and suspicion of it is aroused when a patient has physical signs involving lungs, eyes, skin, lymph-nodes, or spleen. Trouble in any one of these systems in a patient with undiagnosed neurological disease should prompt the physician to embark on diagnostic procedures. Clinical confirmation is most easily obtained by chest radiography and slit-lamp examination of the eyes. Estimation of calcium levels in serum and urine is nowadays regarded as worth while to detect abnormal calcium metabolism. The Kveim test is far more helpful than the Mantoux test and in addition may spare the patient needless investigations, including negative biopsy results. The value or otherwise of giving corticosteroids by mouth will remain in doubt \footnotetext{
${ }^{1}$ Silverstein, A., Feuer, M. M., and Siltzbach, L. E., Arch. Neurol.,

2 Matthews, W. B., f. Neurol. Neurosurg. Psychiat., 1965, 28, 23.

3 International Conference on Sarcoidosis, Amer. Rev. resp. Dis., 1961 , 84, 171 .
}

until the results of a carefully controlled trial are available. Matthews administered corticosteroids to all eight patients in whom the diagnosis was made in life, but it is difficult to assess their efficacy in a disease in which spontaneous resolution is common. The best view at present is the one with which Siltzbach and his colleagues conclude their article. In the absence of any other effective treatment a trial of corticosteroids is recommended for sarcoidosis affecting the central nervous system and for disabling neuropathies of the cranial and peripheral nerves.

\section{Pancreatitis in Childhood}

Starting as an inflammatory process of the ducts and acini, acute pancreatitis progresses to acute pancreatic oedema. Activation of its enzymes by infection or by bile leads to digestion of the pancreas and the process goes on to gangrenous or haemorrhagic pancreatitis. If the patient survives this acute attack he may suffer chronic relapsing pancreatitis. Classically a patient with this condition is a stout-built, hard-living, middle-aged man, frequently with gall-bladder disease and often addicted to alcohol.

In children pancreatitis is usually held to be rare. It most commonly appears as a complication of the exanthemata, particularly mumps, but then the diagnosis tends to be a clinical one without biochemical confirmation. R. H. Dobbs in 1935 published a case of acute haemorrhagic pancreatitis in a 12-year-old girl, ${ }^{1}$ and he found that fourteen cases had previously been reported. Most reports since then have been of single cases: G. B. Stickler and R. H. Yonemoto ${ }^{2}$ reviewed the literature in 1958, and of 38 cases found that seven were due to trauma, one to a trichobezoar, and that three occurred during corticosteroid therapy. Two cases were possibly due to ascarides, with roundworms in the pancreatic duct, and one to mumps, but the cause of the majority-24 in number-was unknown.

Recurrent pancreatitis in children is even less common than the acute disease. W. J. Warwick and R. Leavitt ${ }^{3}$ found only four previously reported cases when they described a single case in 1960. W. H. Hendren and his colleagues ${ }^{4}$ have recently reported the largest series of personally observed cases. Seven children presented as chronic relapsing pancreatitis, and of the eight who presented as acute pancreatitis three developed pseudocysts, and thus the disease became chronic. All survived, and it would seem that pancreatitis in children, particularly in its chronic relapsing form, is more common than generally appreciated.

Acute haemorrhagic pancreatitis is usually a fulminating disease and likely to be diagnosed either pre-operatively or at laparotomy ; milder cases of acute pancreatitis certainly occur and may well be missed unless looked for. The possibility of acute pancreatitis should always be considered in children with acute upper abdominal pain and as a sequel to severe trauma to the upper abdomen. The diagnosis of chronic relapsing pancreatitis is even more difficult, but Hendren and his co-workers suggest that it should be con-

\footnotetext{
${ }^{1}$ Dobbs, R. H., Lancet, 1935, 2, 989.

2 Stickler, G. B., and Yonemoto, R. H., Amer. F. Dis. Childh., 1958, 95, 206.

3 Warwick, W. J., and Leavitt, R., ibid., 1960, 99, 648.

Hendren, W. H., Greep, J. M., and Patton, A. S., Arch. Dis. Childh.,
1965, 40, 132.
} 
sidered in all cases of chronic and recurrent upper abdominal pain. Positive diagnosis is difficult; the levels of amylase and lipase in the serum may or may not be raised, and radiography after a barium meal may or may not show distortions produced by a pancreatic pseudocyst. The morphine-neostigmine provocative test may be of value in children. This test is based on inducing contraction of the sphincter of Oddi with morphine and stimulating pancreatic secretion with neostigmine. It may produce a rise in the serum-amylase level and induce the upper abdominal pain which the child has previously experienced. Hendren and his co-workers carried out the test in six cases. In four it was abnormal, while in the two children with normal results one had no further trouble and the other was found to have no stenosis of the ampulla of the pancreatic duct. Hence the main value of the test would appear to be in the diagnosis of relapsing pancreatitis due to obstruction of the pancreatic duct, which can be treated surgically.

In mild cases of acute pancreatitis laparotomy is not necessary and treatment is supportive. In more severe cases laparotomy may be needed to confirm the diagnosis, and in the very ill child drainage of the lesser sac may prevent the formation of a pseudocyst.

Treatment of chronic relapsing pancreatitis is more difficult. Pseudocysts require excision or some form of drainage, and the gall-bladder may require removal or drainage in the child with congenital spherocytosis. Nevertheless, in $60 \%$ of cases the aetiology of the condition is unknown. It is in these cases that the morphine-neostigmine provocative test may indicate the presence of obstruction to the pancreatic ampulla, which Hendren and his co-workers say demands transduodenal cxploration of the ampulla of Vater and perhaps division of the sphincter.

\section{Causes of Guillain-Barré Syndrome}

The eponym Guillain-Barre syndrome is often given to the type of acute polyneuropathy which is also called acute infective or acute toxic polyneuropathy. In the course of a few days severe weakness of the limbs develops in association with hypotonia and disappearance of the tendon jerks. Sensory impairment is usually less than motor, but there is frequently some loss of proprioception and occasionally of cutaneous sensation. Bilateral facial weakness and bulbar palsy affect some patients, and it is the bulbar palsy which is the most common cause of death if it is not adequately treated. G. Guillain, J. A. Barré, and A. Strohl ${ }^{1}$ in their description of the disease in 1916 stressed that the protein in the cerebrosp nal fluid was much increased without any increase in cells. An exactly similar clinical picture may occur with a normal level of protein in the cerebrospinal fluid, and it seems probable that the protein content depends on how far the spinal roots are involved in addition to the peripheral nerves. If the patient survives the acute stage of the disease complete recovery usually occurs, but in a minority of cases there may be some residual weakness of the limbs. A febrile illness of ten precedes this condition, and the syndrome is thought to be due either to a primary virus infection or to an allergic response in the peripheral nervous system resulting from a preceding illness, usually a virus disease.

Because of the supposed allergic causation steroids have been extensively used in the treatment of the disease, and recently K. N. V. Palmer ${ }^{2}$ has reported a case ending in recovery after treatment with a cytotoxic drug (6-mercaptopurine), given on the grounds that drugs of this type suppress hypersensitivity reactions. The syndrome may follow known virus infections, in particular glandular fever, ${ }^{3}$ and cases have also been reported following chicken-pox ${ }^{4}$ and herpes zoster. A. M. G. Campbell ${ }^{6}$ has suggested a connexion between the disease and feline enteritis after seeing six patients in whom the onset of the disease was apparently directly related to an illness in a cat. J. Marshall, ${ }^{7}$ in reviewing 35 cases, found no clear seasonal incidence except that cases were commoner in the first half of the year. Eleven gave a history of a preceding illness, including respiratory infection, gastrointestinal upset, pyrexia of uncertain origin, and trigeminal herpes zoster. Twenty-one patients denied any preceding illness and from three no information was available. S. C. Melnicks studied 38 patients with the syndrome and found that half of them had circulating complement-fixing antibodies to nervous tissue. $\mathrm{He}$ also considered that the syndrome has features in common with "experimental allergic neuritis" of animals.

Recently Melnick and T. H. Flewett ${ }^{9}$ in Birmingham have studied 52 cases in relation to a possible viral cause. They attempted to isolate viruses from the faeces, throat swabs, and cerebrospinal fluid, and tested the patients' sera for complement-fixing antibodies to a variety of virus antigens. From all except two patients sera were collected on at least two occasions at an interval of seven to ten days. They also studied in detail the incidence of preceding infections and the patients' possible contact with a sick animal. In only two instances was a virus isolated-in one patient from the cerebrospinal fluid and in the other from throat washings. It was Coxsackie B5. One of these cases was somewhat atypical in that there was a lymphocytosis in the cerebrospinal fluid, and as there was at the time a high prevalence of Coxsackie B5 among the general population in the area the authors did not think this finding was significant.

The incidence of cases was more frequent in the first half of the year, especially in February and June. Thirty-six of the 52 patients had had an antecedent illness, including 26 with an upper respiratory infection, 3 with glandular fever, 2 with herpes zoster, and 2 with chicken-pox. These findings were compared with a control series, and when a limit of four weeks before the onset of polyneuritis was taken upper respiratory infections were found to be much more common in the patients than in the controls. The sera of these patients in comparison with those from control subjects showed a high incidence of antibodies for glandular fever and psittacosis, but no higher incidence for influenza $\mathrm{A}$ and $\mathrm{B}$, herpes simplex, or mumps. No patient gave a history of contact with a sick cat.

The authors' findings give no evidence that one specific virus is the cause of the Guillain-Barré syndrome but support the view that the disease may follow soon after a virus infection.

\footnotetext{
Guillain, G., Barré, J. A., and Strohl, A., Bull. Soc. méd. Hôp. Paris, 1916, 40. 1459 .

Palmer, K. N. V., Lancet, 1965, 1, 733.

Peters, C. H., Widerman, A., Blumberg, A., and Ricker, W. A., Arch. intern. Med., 1947, 80, 366.

Welch, R. G., Arch. Dis. Childh., 1962, 37, 557.

Knox, J. D. E., Levy, R., and Simpson, J. A., F. Neurol. Neurosurg. Psychiat., 1961, 24, 167.

Campbell, A. M. G., Proc. roy. Soc. Med., 1958, 51, 157.

Marshall, J., Brain, 1963, 86, 55.

Melnick, S. C., Brit. med. F., 1963, 1, 368.

-395 and Flewett, T. H., F. Neurol. Neurosurg. Psychiat., 1964, 27,
} 